\title{
Métodos de estudio
}

\author{
Study methods
}

Sandra Luz Hernández Mendoza ${ }^{a}$, Danae Duana Avila ${ }^{b}$

\begin{abstract}
:
Currently studying is a conscious and voluntary personal activity that leads to the use of learning techniques that can be very useful for the acquisition of new knowledge, where the study methods are considered as a learning system with which aims to rationalize memory and make the teaching-learning process productive, the application of these methods is important since it corrects certain habits that allow improving or reinforcing academic performance substantially and strengthening very powerful stimuli. These didactic-pedagogical tools are very useful when studying, it also helps us to speed up our mind and have a good memory for the future.
\end{abstract}

\section{Keywords:}

Study, learning, study techniques, education.

\section{Resumen:}

Actualmente el estudiar es una actividad personal consiente y voluntaria que con lleva a el uso de técnicas de aprendizaje que pueden son de gran utilidad para la adquisición de un nuevo conocimiento, donde los métodos de estudio son considerados como un sistema de aprendizaje con el cual se pretende racionalizar la memoria y hacer productivo el proceso de enseñanza aprendizaje, la aplicación de estos métodos es importante ya que corrige ciertos hábitos que permiten mejorar o reforzar el rendimiento académico sustancialmente y fortalecer estímulos muy potentes. Estas herramientas didáctico-pedagógicas son de gran utilidad al momento de estudiar, también nos ayuda a agilizar nuestra mente y tener una buena memoria para futuro.

\section{Palabras Clave:}

Estudiar, aprendizaje, técnicas de estudio, educación.

\section{Introducción}

Hoy en día el estudio es una actividad esencial para todo ser humano donde el rendimiento intelectual $y$ académicos se han convertido en una necesidad social donde en la mayoría de los casos la falta hábitos y la utilización de unas técnicas con un buen método de estudio con llevan al fracaso escolar [1].

Por lo anterior los métodos o técnicas de estudio deben tener un enfoque eminentemente preventivo, debe ser global en la medida que se debe dirigir a los estudiantes, donde la importancia de la aplicación de estos métodos reside en la relación de enseñar y/o compartir experiencias educativas que conlleven a enriquecer el proceso educativo.
Las técnicas de estudio configuran una metodología guiada de la manera de estudiar, las pautas a seguir en el proceso de enseñar.

Cabe mencionar que estas técnicas o métodos ayudan a mejorar y a rentabilizar el estudio; no son, en modo alguno, infalibles, ni tampoco imprescindibles para todos.

Cada persona deberá, en todo caso, primeramente, conocerlas, y, después, elegir aquellas que mejor se adapten a su forma de aprender y retener los contenidos, e, incluso, adaptarlas o inventar nuevas maneras de llevarlas a la práctica. Lo importante es que nos sea útiles, que sirvan para aprender mejor [2].

Por otra parte, estos métodos requieren de hábito, un ambiente adecuado de estudio que permita a cada una de

\footnotetext{
${ }^{a}$ Profesor-Asignatura del Instituto de Ciencias Económico Administrativas. Universidad Autónoma del Estado de Hidalgo. Área Académica de Comercio
} Exterior-Campus ICEA-La Concepción, km 2.5, San Juan Tilcuatla Municipio de San Agustín Tlaxiaca Hidalgo. C.P. 42161. https://orcid.org/0000-00022022-3135, e-mail: sandrahm@uaeh.edu.mx

b Profesor-Investigador del Instituto de Ciencias Económico Administrativas. Universidad Autónoma del Estado de Hidalgo. Área académica de Administración--Campus ICEA-La Concepción, km 2.5, San Juan Tilcuatla Municipio de San Agustín Tlaxiaca Hidalgo. C.P. 42161. https://orcid.org/0000-0003-2286-2843, e-mail: duana@uaeh.edu.mx 
las personas involucradas en el proceso de enseñanzaaprendizaje, superar de manera exitosa los contenidos de aprendizaje, así como adquirir un conocimiento constructivista.
[1] Cortázar, L. (2011). Métodos de estudio. Centro de desarrollo empresarial y de emprendimiento. Politécnico Costa Atlántica. Recuperado y consultado el 19 de enero de 2019 de: https://www.academia.edu/4408667/La importancia de los me todos de estudio.html

[2] Rivera, N. (s.f). La importancia de los métodos de estudio. Recuperado y consultado el 4 de febrero de 2019 de: https://www.academia.edu/4408667/La importancia de los me todos de estudio.php

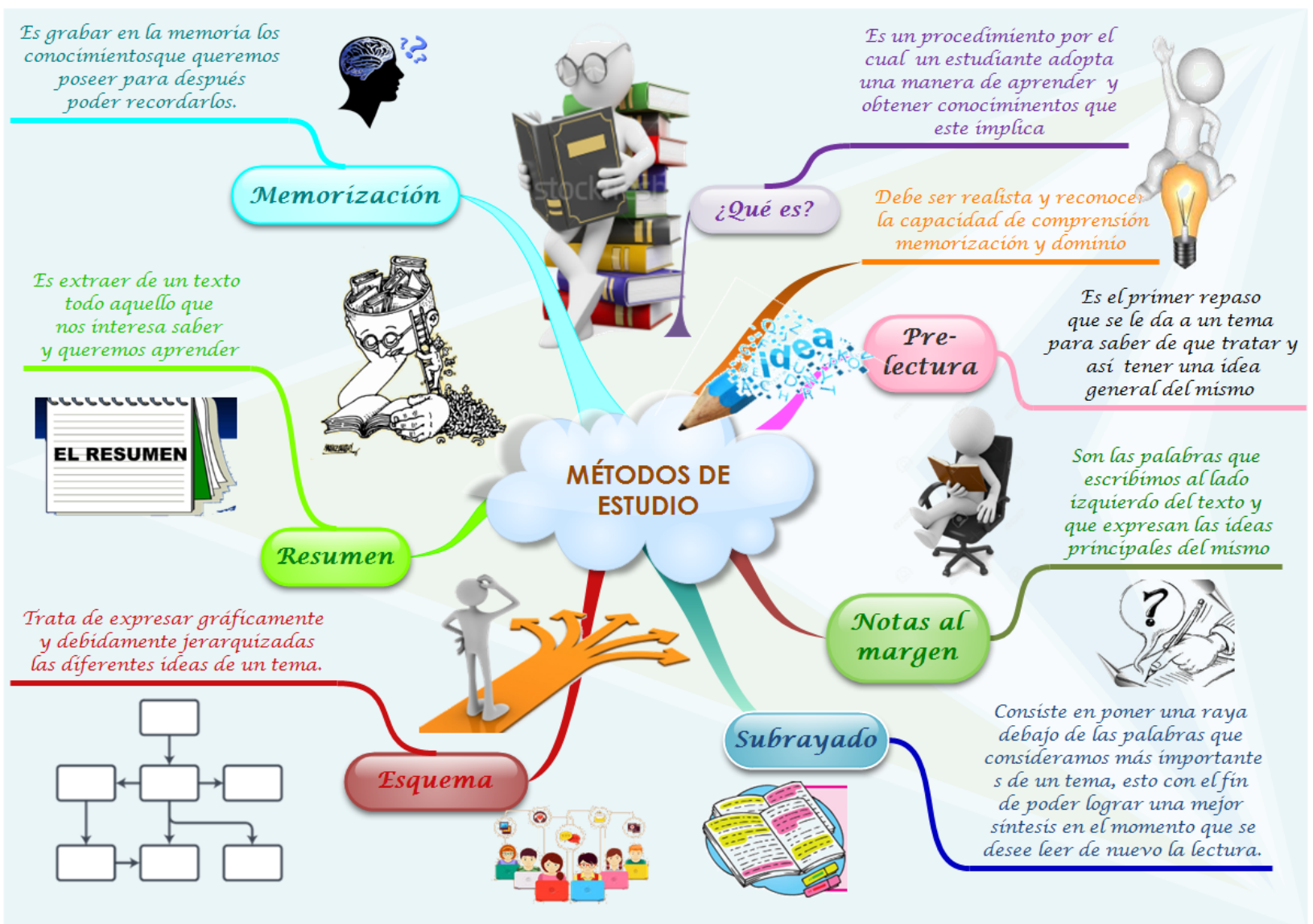

